Portland State University

PDXScholar

Fall 2020

\title{
The Experience of Family Therapists Providing an In- person Group Intervention, Responding to the COVID-19 Pandemic, and Attempting to Continue with a Comparable Intervention in a Virtual Setting
}

Miranda R. Gab

Portland State University

Follow this and additional works at: https://pdxscholar.library.pdx.edu/honorstheses

Part of the Marriage and Family Therapy and Counseling Commons, and the Psychoanalysis and Psychotherapy Commons

Let us know how access to this document benefits you.

\section{Recommended Citation}

Gab, Miranda R., "The Experience of Family Therapists Providing an In-person Group Intervention, Responding to the COVID-19 Pandemic, and Attempting to Continue with a Comparable Intervention in a Virtual Setting" (2020). University Honors Theses. Paper 938.

https://doi.org/10.15760/honors.961

This Thesis is brought to you for free and open access. It has been accepted for inclusion in University Honors Theses by an authorized administrator of PDXScholar. Please contact us if we can make this document more accessible: pdxscholar@pdx.edu. 
The experience of family therapists providing an in-person group intervention, responding to the COVID-19 pandemic, and attempting to continue with a comparable intervention in a virtual setting.

by

Miranda Gab

An undergraduate honors thesis submitted in partial fulfillment of the requirements for the degree of

Bachelor of Science

in

University Honors

and

Psychology

Thesis Adviser

Jennifer Hoskins

Portland State University 


\begin{abstract}
Group interventions are widely used in mental health and educational settings. Studies have shown that these groups can be conducted in either in-person or virtual settings with success, though there is limited research on the delivery of synchronous, online group interventions, particularly an in-person group intervention, that abruptly transitioned to telehealth delivery due to the coronavirus. This observational research aims to tell the story of how two family therapists, who served as co-facilitators for an in-person parenting intervention, transitioned to deliver the same content virtually due to the restrictions of the COVID-19 pandemic. Drawing upon previous and present research in the field, this thesis will connect what the family therapists learned from their direct experience of delivering a group intervention in a telehealth setting and discuss specific ways that the knowledge and implementation of telehealth formats can be strengthened.
\end{abstract}

Keywords: telehealth, group intervention, caregivers, pandemic, Incredible Years, family therapists, co-facilitation, parent, virtual 


\section{Table of Contents}

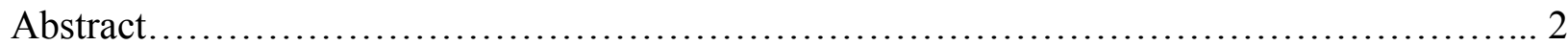

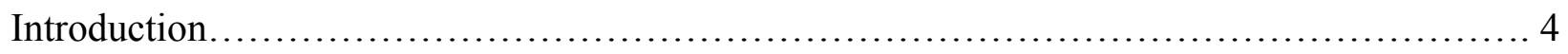

Literature Review

Literature Review Introduction................................................ 9

Modern Considerations Associated with Use of Telehealth Service Delivery........... 10

Assessing Feasibility of a Parent Training Program in a Virtual Setting...................... 12

Evaluating the Comparability of Online Behavioral Parent Training to Traditional

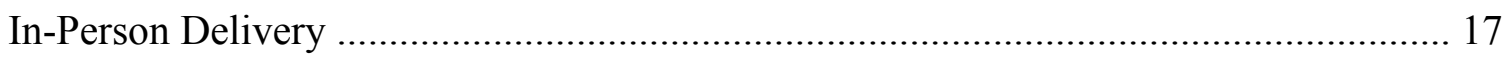

How a Global Pandemic Forced The Exploration of Telehealth Delivery............... 23

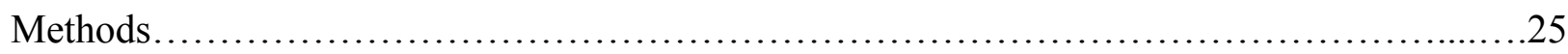

Procedure.................................................................... 29

Outcomes................................................................... 31

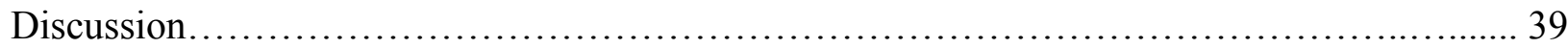

Future Research........................................................ 43

References............................................................... 45 


\section{INTRODUCTION}

Time Magazine reported that roughly $70 \%$ of Americans experienced moderate-to-severe mental distress in the year of 2020, tripling the rates seen two years prior (Heid, 2020). While this cannot be fully attributed to any one cause, the Kaiser Family Foundation conducted a poll which found that $53 \%$ of American respondents reported their mental health being negatively impacted by worry and stress related to the coronavirus, also known as COVID-19. Levels of distress have been rising each month since March 2020, which marked the start of the viral spread in the United States (Nirmita Panchal et al., 2020). Aside from increased stress, the COVID-19 outbreak, characterized as a global pandemic on March 11, 2020 by the World Health Organization (WHO, 2020), brought with it dramatic shifts in day-to-day life for many. This caused a common experience of hardship and heightened concerns regarding the spread of deadly illness, social isolation, financial distress, loss of employment, increased stress, and an indefinite end to the virus. The experience of increased stress during this time resonates strongly with caregivers of children, an inclusive term that will be used in the context of this thesis to describe any individual filling the parenting role. Brown, Doom, Lechuga-Peña, Watamura, and Koppels found in their study that, regarding specific stressors, $21 \%-47 \%$ of caregivers reported that their children had experienced change in both their health and learning directly as a result of COVID-19 (2020). Among caregivers, the most prevalent stressors included changes to mood and general stress levels, with a majority of them experiencing symptoms of anxiety and depression (Brown et al., 2020). While this pandemic has not impacted all individuals, especially 
caregivers, alike, it is important to note that this population is being affected in ways others may not be.

This thesis tells the story of how an in-person group parenting intervention, intentionally designed to address caregivers' stress, was forced to quickly transition to a telehealth model during the COVID-19 pandemic. The following paragraphs will give greater detail to the term telehealth and explain in length how this impacted providers and services for caregivers.

In response to the coronavirus pandemic, various county, state, and federal mandates have restricted capacity for indoor venues, such as places of worship and gyms, to reduce excessive congregation, implemented face covering requirements in attempt to reduce disease transmission rates, and modified business hours for restaurants and bars (The Oregonian, 2020). That being said, one of the most notable and perhaps positive changes is the increased use of telecommunication, which for the purposes of this thesis, will be used to refer to any remote, non in-person communication involving the use of technological platforms. While communication through various technologies has been widely implemented for decades, the novel coronavirus has forced many providers to promptly adapt their in-person care to a virtual style due to social distancing requirements and limited physical contact.

Telecommunication is being used today in the form of phone conferencing, real time video telehealth visits, remote online classes and even virtual group interventions. While some professionals may have experience in telehealth, which for the purposes of this thesis will be used to describe mental healthcare that is delivered remotely by means of technology, many organizations have relied solely on in-person contact. Transitioning to a telehealth platform 
requires hours of additional planning in order to navigate technology. Those with little to no experience conducting their business in a virtual setting may feel resistant and question whether it serves as an equitable replacement. However, for people living in rural or remote areas where services are limited, telecommunication may allow them greater access to distanced providers. Some of the potential challenges for service users are ensuring access to necessary devices, having reliable $\mathrm{Wi}-\mathrm{Fi}$, and the concern of sensitive and confidential information being secure in an online environment (Perle et al., 2011). Additionally, it is important to consider the service user's technological proficiency and whether visual or hearing impairments may pose additional barriers. Fortunately, with the rapid necessity for telecommunication, there are ways to troubleshoot some of these problems and create efficient service delivery.

The dramatic societal shift resulting from the COVID-19 pandemic created an urgent necessity for providers to transition in-person visits to telehealth in order to continue their service delivery. In fact, many healthcare and educational providers are relying on telehealth because of the demands and limitations of the pandemic (Elkbuli, Ehrlich \& McKenney, 2020). Physicians and educators are more frequently employing virtual communications with patients and students via online settings. This allows those in isolation due to coronavirus related concerns to communicate with their respective healthcare or educational teams while retaining the delivery of services (Elkbuli et al., 2020). While there are obvious drawbacks to converting a practice to a telehealth model, there have been several advancements that fill some of those gaps, such as HIPAA compliant service delivery formats and updates to smart devices that allow for improved telehealth accessibility (Elkbuli et al., 2020). Increased utilization of virtual communications can be an innovative way to maintain connections with various other service providers during the 
COVID-19 pandemic. The adapted approach of telehealth is not only limited to one-on-one interactions, but it can be utilized in group settings as well.

One of the communities that could potentially benefit from this uptake in virtual communication is caregivers seeking or participating in parenting support experiences. Parenting groups have been held in various forms, including online internet forums, workshops, mentoring meetings, in-person support groups, and interventions. Each of these settings are appealing to a different audience, and depending on the goals they have in mind, some may fulfill them better than others. Online chat forums may be suitable for the busy, on-the-go caregiver looking for support, and could provide an opportunity to bond and share experiences with others who understand the complexities of parenting. Caregivers who are seeking professional coaching on their parenting techniques may prefer an intervention or workshop where a certified facilitator guides them through various techniques to strengthen their guardian roles and ability to work through challenges. What matters most is that the program they choose is valuable and meaningful to the support they are seeking. For example, caregivers of children with developmental disabilities may find comfort and validation from associating with parents whose children are having similar experiences. Anecdotally, participants from the PRO-Parenting Project, which I will discuss later in greater detail, reported that having shared commonality in their parenting experiences allowed for a deeper sense of connection and belonging in the group. This is an environment that the Incredible Years Parent Training series has been fostering for years. The success of Incredible Years is named here because it is utilized in the PRO-Parenting Project groups, which will be discussed later in this thesis. The Incredible Years series is a program that is designed to promote emotional and social competence and teach ways to reduce 
and prevent aggression and emotional problems in young children 0 to 12 years old (Webster-Stratton, 2001). This program works to reduce children's behavior problems and increase adaptive and effective parenting skills (McIntyre, 2008). Though the Incredible Years series was originally intended to be implemented in face-to-face delivery, it has shown high participant satisfaction, participation and goal attainment in the computer or web-based setting (Taylor et al., 2008). It seems reasonable to assume that with increased implementation of its teachings in virtual environments, this trend is likely to continue.

Several positive outcomes resulted from the transition to telehealth, and the swift adaptations as a result of COVID-19 was transformational. The strength and knowledge acquired through telehealth alone provides innovations that will likely continue beyond the pandemic's time, including the availability and accessibility of services to rural and underserved communities, those with incompatible work schedules and at-risk populations. It also opens up the opportunity to potentially reduce the cost of treatment in some situations, for example, when traveling is involved. Anecdotally, a partnering university to the PRO-Parenting intervention, provided financial assistance for Uber rides and public transportation to families when offering in-person groups. When they transferred to an online setting, these expenditures were greatly reduced. Reducing transportation costs is just one example of the unintended opportunities that resulted from shifting to telehealth. As time goes on, clinicians and researchers will better come to understand the impact of such a sudden and expansive usage of telehealth.

The succeeding literature review intends to focus its attention on parenting intervention groups delivered via telehealth models. While there is limited research on parenting groups for 
guardians of children with intellectual or developmental disabilities (McIntyre, 2008), especially in a virtual setting due to traditionally in-person sessions for these individuals, I intend to share the literature that is available and can be applied in a similar manner to parenting groups. The broad focus of my research aims to tell the story of how a planned 16-week in-person parenting intervention known as PRO-Parenting transitioned to a virtual format due to the COVID-19 pandemic. By closely observing the social workers who served as family therapists that delivered the intervention, I carefully documented their processes and the lessons they learned in shifting to a telehealth model. It is my intent with the following review to showcase the effectiveness, strengths, weaknesses, areas of improvement and key considerations in telehealth delivery as it applies to relevant scholarship.

\section{LITERATURE REVIEW}

\section{Introduction}

This literature review analyzes several publications in order to accomplish a few different aims. The first is to identify the various considerations that need to take place when delivering services in a telehealth format. And secondly, relating these considerations to other studies that have utilized telehealth, exploring successful adaptations and barriers encountered. In the final section, I focus on Dr. Carolyn Webster-Stratton's evidence based curriculum, The Incredible Years. Again, information on Incredible Years and Dr. Webster-Stratton is included because it is the model utilized by the family therapists I observed. In particular, I will review a blog post written by Dr. Webster-Stratton herself, just earlier this year, which highlights her shift in thinking due to the COVID-19 pandemic and the ways she now believes the Incredible Years 
curriculum, traditionally delivered as in-person intervention, can be modified for a telehealth setting, perhaps becoming a permanent delivery alternative.

\section{Modern Considerations Associated with Use of Telehealth Service Delivery}

Perle, Langsam, and Nierenberg (2011) highlighted the debate regarding the integration of computers, the internet and other technologies into therapeutic techniques through review of relevant literature. The article's authors identified the history of non face-to-face therapy, dating back to Freud's practices, where he used letters as an active, indirect therapy (Perle et al., 2011; Freud, 1955). With the progressive increase in the use of technology and interest in adaptation of services to be offered in a telehealth format, Perle and colleagues addressed various considerations that service providers, deliverers and psychology as a field should keep in mind regarding telehealth.

The first consideration was ensuring that services were using the correct term to define their online therapy delivery. Telehealth, as defined by Dielman and his colleagues (2010), is “.... the transmission of images, voice and data between two or more health units via telecommunication channels, to provide clinical advice, consultation, education and training services." Under this umbrella as well, is the use of telecommunications in order to provide assessment, diagnosis, intervention, consultation, supervision, education and information across distance (Dielman et al., 2010). The term 'telepsychology' however, is understood as the "provision of non face-to-face psychological services by distance communication technology such as telephone, email, chat and videoconferencing" (Dielman et al., 2010). While they seem very similar, telepsychology is a subcategory which defines a more specific realm of 
telecommunication that involves the use of other forms of communication (e.g., telephone) in addition to the computer format (Perle et al., 2011). Being mindful of these definitions is important when categorizing service delivery since one term may be more relative than the other.

Another component to recognize regarding telehealth delivery is the treatment modality. It is important to choose a format that is both suitable for the type of service being delivered and for the clients (Perle et al., 2011). Choosing a mode transmission that is either asynchronous, indicating a lag or delay between contacts or content delivery, or synchronous, implying concurrent content delivery, can help narrow down that modality. For example, some providers may find that their services can be delivered with a lag between contact, therefore they may decide to communicate via chat rooms, whereas another provider whose services require real time communication may prefer to use audio or videoconferencing. Both methods are employed by therapists, so finding the best match for the client, service provider and treatment goals is crucial (Perle et al., 2011).

Given the accessibility of technology, telehealth serves as a practical way to communicate with clients. As stated in the text, the majority of people nowadays have access to the technology necessary for an online delivery format, including telephones, smartphones, tablets, laptop computers, desktop computers, Wi-Fi and more (Perle et al., 2011). Use of a telehealth model may also allow service delivery to populations otherwise less able or unable to receive services in an in-person setting, for reasons anywhere from health conditions to transportation access (Perle et al., 2011). The key to utilizing this virtual model successfully is to have a competent provider who is familiar with the content being delivered, the modality it is 
being provided through, and to have the ability to successfully troubleshoot any errors they may encounter.

Because there are no recognized standards for the delivery of telehealth services (NASW, n.d.), it is very important for practitioners to have adequate training and a well-developed understanding of its intricacies. According to the National Association of Social Work, providers should be competent in their work (n.d.), as well as the way in which they are delivering it. This means that a provider must educate themselves in the details of telehealth practices before instating their own practice (Perle et al., 2011). Organizations such as the American Psychological Association (APA) and the Telemental Health Institute that offer training and continuing education credits to those interested in the implementation of telehealth modalities (Perle et al., 2011; APA, n.d.). This provides an avenue to gain knowledge on the operation of telehealth models, efficient delivery, familiarization with the function of the platform used, and best practices for serving clients. An additional benefit to taking these courses is that they can inform providers regarding how to maintain confidentiality and security in their delivery. This is vital for any therapeutic setting, but perhaps more so in an online platform.

While Perle's article identified essential factors to consider when using telehealth platforms, the next article written by Bearss and colleagues narrowed in on assessing the feasibility of delivering parent training via telehealth.

\section{Assessing Feasibility of a Parent Training Program in a Virtual Setting}

Bearss, Burrell, Challa, Postorino, Gillespie, Crooks, and Scahill (2017) piloted a telehealth study to assess the feasibility of a 24 -week parent training program developed 
specifically for parents of children with Autism Spectrum Disorder (ASD) or a disruptive behavior. It was intended for this delivery to serve as a potential solution to the limited access of specialized service delivery, which in this case is parent training, specifically for children experiencing ASD and disruptive behavior. Aside from the goal of delivering valuable services to these families in general, a key motivation for this study was to expand the pool of available service options (Bearss et al., 2017). This project was delivered in a clinic-to-clinic telehealth fashion, meaning that the clinicians delivered the parent training from one clinical treatment center via a telehealth platform to the clients, who were in another clinic over 180 miles away on average (Bearss et al., 2017). Throughout the course of this program, researchers tracked a multitude of measures. The results of the study show support for the feasibility and preliminary efficacy of the parent training program for this target group when delivered in a telehealth setting, achieving rates of $90 \%$ or better in the following categories: completion, attendance, attainment of subject objectives and fidelity (Bearss et al., 2017). While there is still further research needed in order to test whether parent training via telehealth is a viable way to extend this evidence-based treatment, Bearss and colleagues' research paved the way with promising results which support the PRO-Parenting study's transition to telehealth.

Relating Bearss' study to the considerations presented by Perle and colleagues, a majority of the key points were addressed. The first is that they were able to identify their program as "telehealth." The use of this umbrella term gave their study a bit of flexibility in defining their model, as it was not as specific or fitting as a term like "telepsychology." 
Additionally, they identified a suitable treatment modality for their program. The program was delivered through synchronous, or real-time, communication, in which the clinician and client met via videoconference in their respective locations. This was a delivery suitable for the clients and their needs. Due to the distance from client's residences to the providers, meeting in the middle at a clinic location was an adequate alternative (Bearss et al., 2017).

This study also ensured another consideration proposed by Perle's article, which is the accessibility to technology necessary for a telehealth delivery format. As Perle states, the majority of people have access to Wi-Fi as well as a device to use for telehealth delivery (2011), however that still leaves some individuals without access. To combat this potential issue, Bearss and colleagues chose to use a clinic-to-clinic model, increasing the reliability of the access to and the performance of the technology used. Performance of technology was a concern in this particular case because the clients were from rural areas where there was the possibility of unstable connectivity that may not be as prevalent in other areas. Additionally, having clients present in the clinic while the program is being delivered allowed for present support staff at the clinic sites to help address any technical issues that the clients may have encountered during their videoconference.

Perle's final consideration was the absolute necessity of the practitioner's training and understanding of telehealth in order to conduct online groups. As stated previously, there are no standards for telehealth (APA, n.d.), therefore it must be practiced with the utmost care, intention and thoughtfulness. The four clinicians in the study received training on the content they were going to be delivering prior to the start of this project (Bearss et al., 2017). However, there is no 
indication as to whether or not the clinicians received training in telehealth prior to the study taking place. While it is implied that the support staff at the clinic sites were knowledgeable in telehealth technology, it was not explicitly stated they had any additional skills or training. Just as it is important for service providers to be educated in the content they deliver, being skilled in the platform they choose to utilize is also crucial. Continued education courses are available for any provider who is operating in a telehealth setting and desires to improve their skills. Anecdotally, the PRO-Parenting family therapists who I observed for this thesis have gone on to regularly meet with other service providers who are also using telehealth. The purpose of these meetings is to expand their knowledge of virtual modalities so that the telehealth experience does not impede the delivery of evidence-based content to service users.

Bearss' research aligned with many of the suggestions outlined by Perle and his colleagues. Their study also shares distinct similarities to the PRO-Parenting intervention, yet there are still areas for improvement. Beginning with the strengths, their study provided services to families with high needs that otherwise may have not been served due to living in rural areas. Implementation of the program was also effective in this study, as can be seen in the results. There were high rates of completion (97\%), attendance of core sessions (92\%), attainment of subject objectives (95\%), and fidelity (98\%), modeling this study as rather feasible (Bearss et al., 2017). The study's successes can be attributed to their practice, maintenance of fidelity to that manual, and multiple measures to assess various components of the study.

More critically, however, this study also had some deficits. This was a small sample of only 14 participants and no control group. Without the presence of a control group, it is 
challenging to differentiate the effects of treatment from other impressionable factors, such as the effect of time or attention on disruptive behavior in children with ASD (Bearss et al., 2017). Bearss' research did not explore the idea of participants accessing content via their own homes and through use of personal technological equipment. Because this study was conducted far before the COVID-19 outbreak, it is unreasonable to criticize them for not considering alternative delivery formats. However, with the many restrictions put in place due to the pandemic, the possibility of, for lack of a better term, "home-to-home" delivery, would be optimal. With that being said, providers delivering treatment from their home to clients in their home in a telehealth environment is not a far-fetched idea. While certain factors could impede this delivery model, such as limited access to the internet or need for increased funding to support participant access to necessary technology, given the restrictions of the pandemic, it deserves more exploration.

This article was able to show Perle and colleagues' suggestions carried out in practice, demonstrating that they are helpful and can provide a realistic blueprint for designing telehealth groups. Bearss et al. aligned in key areas with Perle and colleagues. The succeeding paragraphs will look at work done by DuPaul and colleagues, who created a study that utilized a behavioral parent training program for parents of children with or at risk for Attention-Deficit Hyperactivity Disorder (ADHD) in order to evaluate the extent to which the program was feasible, acceptable and efficacious in an online setting. 


\section{Evaluating the Comparability of Online Behavioral Parent Training to In-Person Delivery}

The most commonly researched and efficacious psychosocial intervention for young children experiencing ADHD is parent education through the use of behavioral parent training (DuPaul et al., 2017; DuPaul \& Kern, 2011). DuPaul, Kern, Belk, Custer, Daffner, Hatfield, and Peek developed this pilot randomized control study to examine parent engagement and program acceptability of face-to-face and online behavioral parent training (BPT) geared towards families with children at risk for or diagnosed with ADHD (2017). The efficacy of both formats was also assessed relative to a waitlist control group (DuPaul et al. 2017). Motivations for this study lie in the lack of community access to BPT (DuPaul et al., 2017). Since BPT is typically delivered in face-to-face settings, there are limitations set in place, given that individual circumstances may prevent families from being able to attend in-person interventions. Their study aims to assess whether a BPT intervention can be comparably conducted in an online setting, offering a potential increase in accessibility that is not widely present at this time.

The overall purpose of this study, as stated by the authors, is to examine the degree to which a 10-session BPT program designed to target behavioral concerns and needs specific to those with or at risk for ADHD would impact parent engagement, parent stress, and child behavior. The participants in the study were randomly assigned to participate in either the face-to-face program, the online program, or they were placed in the waitlist control group where they would receive treatment after the conclusion of the face-to-face and online intervention delivery (DuPaul et al., 2017). 
The face-to-face participants attended 10 consecutive live sessions at a local school, instructed by graduate-level students in school psychology or special education (DuPaul et al., 2017). Childcare and food were provided to the families during the intervention sessions (DuPaul et al., 2017). After the end of each session, the interventionist and other research team members held a debrief session where they reviewed the delivery of content, participation of parents in group discussions and any questions the interventionists had about the treatment procedures (DuPaul et al., 2017). The participants assigned to the online program were required to attend the first session in person along with the face-to-face participants to ensure they could successfully $\log$ into the program (DuPaul et al., 2017). The following sessions were conducted asynchronous in an online format. New sessions were released weekly and remain open and accessible throughout the course of the intervention, allowing participants to complete any of the sessions from the duration of the program (DuPaul et al., 2017). The research assistants checked in via phone call with each of the online participants in between the release of sessions, to check in on their progress with the intervention as well as address any questions or concerns they had with the curriculum. Additionally, the online program tracked progress of each participant throughout the intervention (DuPaul et al., 2017).

The results of this study showed many positive outcomes. Both treatment conditions, the online and face-to-face delivery, were associated with high rates of parent engagement, where $80 \%$ of the treatment sessions were completed on average across the two conditions (DuPaul et al., 2017). High levels of engagement in the online delivery could be contributed to the weekly check ins conducted in between sessions, more or less promoting the parents to follow through with the weekly tasks (DuPaul et al., 2017). Comparing both the face-to-face and online groups, 
attendance was higher in the face-to-face program which could have been due to a number of factors, including the provision of food and childcare services to the in-person families. The online BPT program involved less interaction amongst parents, therefore it was noted that this could have led to higher session completion rates in the online delivery (DuPaul et al., 2017). DuPaul and colleagues do not further elaborate on the reasoning behind this assumption, but speculate that it may have to do with fewer distractions in the asynchronous online format (2017). The two BPT conditions ranked high in knowledge attained by parents and fidelity with regards to the intervention strategies (DuPaul et al., 2017). Furthermore, both the face-to-face and online delivery were ranked at least moderately acceptable by the parents using the Intervention Rating Profile-15 (DuPaul et al., 2017). DuPaul and his colleagues stated that the online parent training led to significant changes in child behavior that was comparable with results typically found in face-to-face behavioral parent training (2017). This indicates that while it may not be a perfect alternative for in-person intervention delivery, it is a commensurate delivery option and has strengths that are unique to its format. Lastly, improvement in parents' knowledge and treatment fidelity were accompanied by a decrease in parental stress for both groups, which was one of the items researchers tracked regarding the success of the program and its delivery.

This study, however, did have its limitations. The first is that it was a pilot trial with a small sample size. This limits the statistical power that these results hold (DuPaul et al., 2017). Secondly, since behavior improvement was based solely on the parent report, this may open up an opportunity for biased results, given that the parents were directly involved in the delivery of the intervention. This could have been avoided with the use of behavioral evaluations from an 
external source (e.g., a teacher, day care provider) or an in-home observation (DuPaul et al., 2017). Another limitation of this study is while they did see a decrease in challenging behaviors among the children with or at risk of ADHD, their behaviors post-intervention were still in the critical range (DuPaul et al., 2017). Essentially, this finding indicates that the treatment didn't "normalize" the children's behavior, it just aided in behavior improvement (DuPaul et al., 2017). Finally, there is a possibility that these findings have low generalizability because of the relatively high education of the parent participants in the study. Although the parent sample was diverse in terms of race and ethnicity, their higher socioeconomic status (SES) and education level could have impacted engagement, parent knowledge and other factors.

Connecting this back to Perle and his colleagues' article, this study also addressed many of the key considerations that they presented. Regarding the definition of their online delivery, DuPaul et al. characterized their format very loosely. It is referred to in the text as "online program," unlike Bearss and colleagues' use of the term “telehealth" (DuPaul et al., 2017; Bearss et al., 2017). Their lack of specificity, as I interpret it, tells me two things. First, it rids them of any association between their project and healthcare, as indicated by the term "telehealth", which may misrepresent the work that they were attempting to achieve. Building off of that, it also tells me that it is possible there is a need for development of other terminology that is more suitable for programs like this one.

In terms of treatment modality, this study took on an asynchronous delivery for their online setting. This choice highlighted their goal of increasing accessibility to families. The "at 
your own pace" delivery of this program allowed the intervention to be more flexible for families who may not have otherwise been able to attend the in-person sessions.

In this study, it was part of the inclusionary criteria regarding eligibility that the parent participants had access to reliable internet connection and technological devices in order to participate. This placed the responsibility on the families. A pitfall that accompanies this is that there will be some families who are excluded from the intervention solely because of this criteria. This could leave lower SES families out due to not having access to Wi-Fi or smart devices.

Lastly, Perle and his colleagues recommend that the interventionists need to have adequate training and understanding of the content they are delivering and of the online delivery format. The article mentions that the interventionists were graduate-level students in relevant areas of study, therefore it is likely that they have an adequate knowledge of the program's content. However, there is no mention of online delivery training. With that being said, I believe that this is because the interventionists of the program were not actively leading the online format. It was an independently-led program where the parent participant was in charge of the delivery. A positive aspect to this, however, is that the online delivery participants were given access to technical assistance in the event that they were to experience any difficulties.

One of the primary reasons that this publication was selected for review is because of its striking similarities to the PRO-Parenting Project, which is the name of the project I observed transitioning to telehealth delivery. The PRO-Parenting Project is an intervention that uses BPT curriculum to guide caregivers of young children with developmental delays through effective 
ways to reduce child behavior problems and caregiver stress (McIntyre \& Neece, 2018). These two studies are complementary in many ways, starting with their composition. Both projects used random assignment to place caregivers of children ages three to five years to either condition. DuPaul and his colleagues randomly assigned participants to be in the in-person group, the online group, or the waitlist group (2017). Whereas the PRO-Parenting Project, as originally intended, randomly assigned participants to one of two in-person dual-intervention groups. One group emphasized education and social connection in the weeks leading up to BPT and the other taught mindfulness practices in the weeks prior to the BPT. Each project had 10 weeks of BPT delivered by master level clinicians, who also conducted weekly check-ins. Both projects were conducted with groups that had higher SES and education of caregivers and they both offered stipends for completion of measures. They also used several similar measurement tools, such as the Developmental Ability Scale (DAS) and the Parenting Stress Index (PSI-4). Amongst these similarities lies a great deal of differences as well. While DuPaul excluded children based on having a developmental delay or Autism diagnosis, the PRO-Parenting study specifically included these children. Additionally, DuPaul's project had a waitlist control group, allowing there to be a baseline point of reference for each of the delivery formats. In terms of measurements, DuPaul's study used some that were specific to their participant focus which was children with or at risk of ADHD and they also tested their parent participants for information retention.

An important takeaway from this comparison is that there is work being done to further develop the field of telehealth. Each of these studies has important implications for future research and have done a great deal of work to fill in the gaps in their discourse community. 
Even though DuPaul's face-to-face participants ranked higher in fidelity and rated the intervention higher the online participants, this should not deduct merit from the achievements of the online group. While there are similarities between the PRO-Parenting Project and DuPaul's study, the PRO-Parenting project was not originally intended to be delivered in an online format. When this became the only option due to the COVID-19 pandemic, the content delivery remained live and synchronous in a virtual format. The intervention model used in PRO-Parenting was adapted from the Incredible Years, a parent training program developed by Dr. Carolyn Webster Stratton. In the next section, the delivery of the Incredible Years model in a virtual setting will be further explored.

\section{How a Global Pandemic Forced The Exploration of Telehealth Delivery}

Dr. Carolyn Webster Stratton developed the Incredible Years parent training program more than three decades ago and still today it remains a highly-ranked, evidence-based curriculum (Webster-Stratton, 2020) In mid-August of 2020, in response to the COVID-19 pandemic, Dr. Webster-Stratton published a blog post telling about transitioning her once solely in-person parent training program into a telehealth model. As she states in the blog, Dr. Webster-Stratton held a strong belief in the importance of in-person interaction and the value of collaborative learning, problem solving, role plays and practices (Webster-Stratton, 2020).

Previous work she had done a few decades prior found that there were better parent and child outcome effect sizes when parents received their training in group interventions with the use of video modeling, as opposed to other methods such as self-led programs (Webster-Stratton, 2020). The thought of delivering these groups in an online environment was unsatisfactory 
because she believed there was no substitute for the experience gained from in-person group settings. However, Dr. Webster-Stratton was forced to consider comparable options for delivery due to the coronavirus outbreak. She found herself, almost without choice, to utilize an online delivery format (2020). Dr. Webster-Stratton states that the technology was intimidating and frustrating to use, likely making an impression on her feelings towards this method of delivery. Once she learned that there were ways that the similarities between the delivery methods could better mirror one another, her perception and outlook on online delivery began to change. In her blog post, she states that "perhaps it is not the platform that makes one training method more effective than the other, rather it is the group leader who is able to make the individual or group feel safe and engaged online" (Webster-Stratton, 2020).

Within this post, Dr. Webster-Stratton provides tips for leading online groups. First, it is important for group leaders to allow themselves to experiment with technology and find ways to incorporate fun, collaboration and meaning into the group (Webster-Stratton, 2020). Next, leaders should lower their expectation of covering the same amount of content in an online session as they typically would in an in-person setting (Webster-Stratton, 2020). Lastly, Dr. Webster-Stratton states the importance of remaining flexible with technological challenges (2020). It is necessary to remember that there is no such thing as seamless execution right off the bat, so be prepared to encounter challenges and choose to learn from them.

Lastly, one of the most impactful outcomes resulting from Dr. Webster-Stratton changing her view on online intervention delivery is openness to offering services to individuals who may not be able to attend traditional in-person groups. This could happen for many different reasons, 
such as lack of transportation, incompatible schedules or other conflicts (Webster-Stratton, 2020). Various functions in the online delivery platform, such as the virtual whiteboard and chat functions, can be used to mimic in-person sessions. This is notable regarding the PRO-Parenting Project because the team implemented multiple online platform functions and developed various ways to make the online groups interactive, creating an experience as similar to face-to-face groups as possible prior to the publication of this blog post.

In summation, Dr. Webster-Stratton found herself in a pivotal moment in history where the best, and perhaps only, option she could take on to continue her Incredible Years program was to go against her prior beliefs that in-person group training was the only efficacious style. Amidst taking the path she was hesitant to travel, she discovered the valuable potential of providing caregivers who experienced a lack or delay in services due to differing constraints (e.g., rural location, lack of transportation, COVID-19 shut down) with an evidenced based parent training that is shown to have positive impact on child behaviors and family outcomes. Being able to document Dr. Webster-Stratton's transformation on this topic is so unique because it is unfolding in real-time. This is an advancement that is going to last beyond the scope of the pandemic.

\section{METHODS}

The key point of departure for this thesis was the PRO-Parenting Project. Partnering the University of Oregon's Prevention Science Institute in Portland, Oregon, and Loma Linda University in Loma Linda, California, the PRO-Parenting Project was intended to assess the benefit of adding a caregiver stress-reduction intervention prior to the delivery of BPT in hopes 
of effectively reducing behavior problems in children with developmental delays by way of increasing caregiver's retention of information using BPT curriculum (McIntyre \& Neece, 2018). The Incredible Years model influenced the BPT technique used in the present research. This curriculum was adapted to be used on parents with children between three and five years of age experiencing developmental delay and/or disability (McIntyre \& Neece, 2018). This intervention guides caregivers through various ways to approach behavior concerns with thoughtful adaptation focused on experiences of children with developmental delays. The PRO-Parenting project, as previously mentioned, was originally intended to be delivered in an in-person setting. However, as I will further explain, due to the COVID-19 pandemic, the intervention was provided in a live virtual format allowing participants to safely access content through the screen of their computer or phone. The family therapists facilitating these groups have an experienced background as social workers.

My position in this project at its conclusion strayed very far from the initial job description. At the start of my journey with the PRO-Parenting Project, I was hired as a childcare worker, where my duties included providing supervision and facilitating play activities with the children of the caregivers who were taking part in the face-to-face intervention group. Unfortunately, as the COVID-19 outbreak began to sweep the globe, each PRO-Parenting team members' role changed in unanticipated ways. The shutdown in Oregon began with the cancellation of all non-essential school-associated gatherings and group activities, which prevented the intervention from being conducted as the sessions were held after hours at a local elementary school (Multnomah County, 2020). This action was followed shortly by the banning of all larger public gatherings, causing schools to shut down and continue their learning in virtual 
platforms (Dake, 2020). As businesses were ordered to limit their services, number of customers or perhaps even close their doors, the Oregon Governor eventually put a "stay home" order in place, eliminating as much close interpersonal contact as possible (VanderHart, 2020). While this meant something different for each organization, for this project, it meant no gathering amongst guardians, therapists, researchers, or families participating in the intervention, therefore terminating the need for a childcare provider. This posed the potential halt of services, which would have left the PRO-Parenting families without the support and the additional benefits that came with their participation in this project. The PRO-Parenting team then had to address the ethical decision of whether to move forward with this intervention.

First, it was important to recognize the lack of skill and experience the team had in delivering groups in an online environment. As stated in the National Association of Social Workers' Code of Ethics, social workers should only provide services within the confines of their education, license, certification or other relevant professional experience (NASW, n.d.). Because the family therapists had never delivered this content virtually, doing so would have been outside of their professional expertise. There was also acknowledgement that virtual delivery does not have recognized standards in this area of practice and, accordingly, the NASW encourages social workers to exercise careful judgement in ensuring they are competent in their work and that they are protecting their clients from harm (NASW, n.d.). Considering the latter factors alone, the lack of experience and knowledge around conducting virtual groups may have been enough to deter some from attempting to continue in this fashion. However, an important counterpoint was posed that carried a great deal of weight. 
Article 1, section 15 of the NASW Code of Ethics states that social workers should make reasonable efforts to ensure the continuity of services in the event that services are interrupted for any number of reasons (NASW, n.d.). A study conducted with both the Portland and California PRO-Parenting cohorts at the beginning of the COVID-19 outbreak found that $72 \%$ of the families experienced delay in early childhood support or education services (Neece, McIntyre, \& Fenning, 2020). And, as mentioned earlier, caregiver participants were screened using the PSI-4 and found eligible for the study based on having heightened stress levels. Meaning that the caregivers involved in this project were already having increased stress, even before the pandemic hit. This information combined with pandemic related parenting stress being reported society wide (Heid, 2020), led the PRO-Parenting team to conclude that study participants may benefit from continuity of care. Because these families had already experienced loss or delay in some or all of their services, the PRO-Parenting team felt that they had an ethical obligation to proceed with the group intervention. While this was a complicated clinical choice, after careful consideration, it was determined that it would be unethical for the team to abandon these already vulnerable families in a uniquely stressful time. Therefore, it made the most sense to make the appropriate steps for the continuation of the intervention. The PRO-Parenting team filed appropriate forms and received approval from the Institutional Review Board at the University of Oregon to shift these groups to a virtual setting, where they would be able to continue provision of services. The team adapted each employees' roles to suit the virtual execution of the project and challenged themselves to continue the sessions in a live virtual format through use of Zoom. This online communication platform allowed for secure, HIPAA compliant group meetings between therapists, project researchers and families, resulting in the 
successful completion of the intervention. My new role in the proceedings of this project was to document the experience of the family therapists as they attempted to continue with a comparable intervention in a virtual setting.

\section{PROCEDURE}

In the new virtualized PRO-Parenting project, I took on the role of an observational researcher. My duty, as this role entails, was to systematically observe and record the proceedings of the project. The first step in doing this was to attend PRO-Parenting team meetings. In these meetings, the family therapists and PRO-Parenting research team came together to discuss, plan, and eventually implement the intricacies of the intervention. The meetings involved conversations on various topics, including discussions regarding the technology being used, how to best serve the participant families through the stresses that COVID-19 may be exacerbated in their lives, how to maintain fidelity as closely as possible to the original design of the group intervention, and constant reflection on what was going well and what areas could be improved upon. Additionally, these meetings occasionally served as accountability check-ins for the project members when tasks were assigned and asked to be implemented or reported on later.

While observing these meetings, I took extensive notes on the conversations that took place, ideas presented by team members, and events that occurred. These notes were dated and time stamped to maintain an accurate record of the proceedings of the meetings and project overall. They served as documentation of the experiences in this dramatic shift to a live telehealth setting as it was experienced by team members and participant families alike. The 
observation and notes were another way to ensure fidelity to the project plan in addition to the research assistants' roles as fidelity checkers during the scheduled group intervention meetings. In efforts to maintain confidentiality and security of the information within the notes, they were taken in a private setting and stored in a locked safe thereafter.

I attended dress rehearsal run throughs of the groups with the family therapists and other project members as well, documenting the topics covered in each week's content. These meetings offered the family therapists the opportunity to practice the material and confer with the California team to ensure alignment of content delivery across sites. In these meetings, they identified potential questions that caregivers could ask and made sure that they delivered the content in the allotted amount of time. During dress rehearsals, team members logged on with their phones and computers to ensure the best user experience of participants. Notes on these dress rehearsals also included any changes that were made to the delivery of information and new features that the family therapists utilized, such as the whiteboard function or issuing virtual rewards for their participation or in response to successes parents were having relative to the curriculum.

After the groups were conducted each week, I attended debrief sessions with the family therapists to address the overall execution of the group sessions. In these meetings, the three of us focused very specifically on what went well, conversations, comments or valuable examples that caregiver participants had in the group session, challenges they may have encountered, and how they either managed those obstacles in the moment or ways they hoped to handle it in a future situation. Amidst this debrief session, I asked the family therapists additional questions to 
gather a more in-depth understanding of the development of the project. Lastly, and perhaps most importantly in the long run, these meetings allowed time for the family therapists to recall and reflect on their experiences from their previous group sessions, aiding in preservation of their lessons learned in this setting.

As this project came to an end, I compiled my notes together to develop common themes that were experienced by the family therapists and other team members throughout this virtual shift.

\section{OUTCOMES}

While observing the family therapists who functioned as group facilitators for this project, we had several conversations regarding the choices they made to conduct these groups in the best fashion. Amidst these discussions, the facilitators were able to identify the most valuable takeaways from the experience and synthesize them into 14 key points. These lessons aim to describe in detail what they found to be successful in executing a group intervention through a telehealth setting.

The first lesson is the importance of having a co-facilitator. It is incredibly helpful to have another person involved in the delivery of these groups. This allows facilitators to switch back and forth between one another when delivering content, maintaining attention of the group participants while keeping the experience interesting. Co-facilitation also allows more opportunities for either facilitator to identify what is happening for the caregivers in the group as content is being delivered (Cresswell, 2014). Additionally, it offers the ability to better cater to caregivers' needs since there are two facilitators to address them (Cresswell, 2014). This can 
help alleviate the responsibility of either facilitator, knowing that they will have a partner in the delivery process. This allows either facilitator to answer questions, provide different perspectives, and potentially continue leading the group in the event the other facilitator encounters technical difficulties or is unable to attend. For example, Loma Linda University’s team encountered a situation where one facilitator got injured. Without hesitation, the other facilitator was able to be the primary group leader that evening. Co-facilitation in this instance prevented the cancellation of the group session. Further, having two facilitators combats the risk of one of the therapists encountering internet outages or technical difficulties, making it difficult for them to stay logged into the group. A second facilitator ensures that the group is never abandoned in a virtual setting.

The next lesson is the importance of having technical support during the groups. This allows the facilitators to focus on leading the group while the technical support person is able to resolve any of the technical issues that inevitably arise. If the facilitators have to stop the groups to handle these challenges, it could disrupt the content delivery and dissolve group members' attention. The technical support is essentially a binding agent that allows the group to run smoothly, handling all issues behind the scenes. In the PRO-Parenting Project, fidelity checkers and research assistants who were sitting in on the group meetings acted as technical support in each session. The facilitators introduced the technical support by name at the beginning of the group, which was then reinforced with the assigned technical person sending a message in the chat informing the group members they were available when needed and adding in their phone number for additional communication and technical support. 
The group facilitators also found it necessary to keep in mind that the virtual setting is performative. Because the group is not gathered together in person, there is a lack of warmth and connection felt among members of the group and its facilitators. Therefore, there must be additional effort put in to display this connection from one screen to another. It can be theatrical at times, but it can make all the difference. These enhanced performative measures can take on many forms. First, it is important to greet people as they enter the virtual space. This recognition assigns importance to each group member's attendance, creates a sense of belonging and generates a space that is welcoming. It is also useful to be aware of the facial expressions that are displayed. Just as one should monitor their body language and facial expressions in person, it should carry over to this setting as well. Be mindful of one's resting face as well as the facial reactions to questions, answers or other statements made by group members. It is also important to be more expressive than usual in a telehealth setting since the camera and microphone cannot always capture the magnitude of emotions. This includes exaggerated hand gestures, looser movement and big smiles. Lastly, all attendees should use the background of the video in a positive way. This means moving away any clutter or distractions present in the background of the video, changing up the art or displays in the room that may serve as conversational pieces, and using virtual backgrounds such as eye-catching landscapes. The goal of this point is to cast focus on the individual in the frame as opposed to their backgrounds.

The fourth lesson is walking group members though Zoom etiquette. Similar to how group rules or expectations are established in the beginning of a series of in-person sessions, this should take place in a virtual setting as well. Teaching group members about the various 
functions of the platform that the program is delivered on will familiarize them with it, as well as show facilitator knowledge and understanding of the platform. During the experience of the PRO-Parenting group, they ended up having to teach the caregiver participants Zoom etiquette along the way as they encountered certain obstacles. For example, after a few instances of audio disruptions from group members, group facilitators encouraged the use of the mute function if the background noise was unmanageable or distracting. Facilitators also chose to use the "mute all" function on Zoom in order to silence all noise that was not part of the content delivery, establishing a group norm. Once this was widely practiced by group members, the facilitators discontinued the use of the "mute all" function and caregivers utilized it on their own. It is important to also remind participants to unmute themselves when they want to talk, otherwise they will not be heard by the facilitators or the rest of the group. One thing to keep in mind regarding the "mute all" function or a group norm of being muted during content delivery is that it could potentially discourage group discussion or answers from participants. By encouraging all group members to unmute themselves when it is time for group discussion, this may be a way to motivate organic discussion.

Lesson five is that group leaders must pay attention to the faces of the participants. Watching their reactions to what is being said in the group allows the facilitator to gauge how the participants are responding to the material. For example, facilitators may notice when a participant wants to speak, such as when they unmute themselves or lean towards the camera. The facilitator can make sure to provide an opportunity for that participant to chime in and make sure they are not accidentally ignored. Another thing that this attention offers is the ability to say things like "I saw a lot of nods there, this seems like a common theme for everyone." It can be a 
way to unite multiple reactions and foster a sense of connection. When leading a group in person, these are all things that leaders would likely exercise, therefore there is no question it should also take place in a live telehealth setting. It is an effective way to conduct the group and establishes the facilitators as perceptive and caring.

Next is that a PowerPoint or other slideshow presentation can work very well in a virtual setting. In an in-person group, PowerPoint presentations or other slideshows may be a bit grueling to pay attention to or it has the potential to take the focus away from what is being said by the facilitators. Inappropriate use of a slideshow can degrade the quality of a presentation, which is not the desirable outcome. In this study, the facilitators found that the PowerPoint functioned as a centerpiece of the group or served as the "dinner table" that they were all sitting around. With that being said, it is important to consider the way that the PowerPoint is used. It should be visually appealing, have a limited number of words, avoid flashy transitions and excessively vibrant colors, and contain images that are complementary to the content being delivered.

Strengthening that last point, lessons seven and eight highlight the importance of using graphics and visuals to keep the presentation lively. The facilitators found that word-heavy slides were tiring for group participants and a barrier for individuals with reading difficulties. Using graphs and cartoon graphics can also be used to create this balance. Additionally, the photos or cartoons used should be diverse and representative of the community of the group. For parenting groups in particular, this could include the display of same sex couples, families of color, mixed 
race families, single-parent families, children with disabilities and many more. This diversification will allow the participants to see themselves in the curriculum and thus relate to it.

Lesson nine is the importance of practice. Rehearsing the presentation of the content is important for several reasons. First, it is crucial for the facilitators to be familiar with the information they will be delivering. Increased competence and understanding offers better content delivery and helps identify how much time is being used on certain sections of the content or the overall presentation if there are time constraints. Practice will also help facilitators master technical transitions. For instance, the Incredible Years series used in the PRO-Parenting Project uses videos in its teachings and, in order to present these videos over Zoom, the facilitator has to toggle between different programs in order to set up and play the videos. Practice sessions allow facilitators to refine this transition so they are smooth when the groups take place. Lastly, facilitators should get a feel for what the group meetings look like for participants using different devices to attend meetings. The groups will not look identical between someone who joins with their cell phone versus someone who joins with their desktop computer. Addressing how different features appear on these devices is an inclusive and thoughtful decision made by the facilitators.

The next lesson is rather specific to the PRO-Parenting Project and their use of the Incredible Years curriculum, but it is valuable nonetheless. In the Incredible Years series, there is a component called Parent Principles, in which the group facilitators take note of wise statements made by parents that highlight their understanding and execution of the curriculum. This is typically done by hanging up the noteworthy quotes or actions around the room, so their space is 
filled with group member accomplishments. This is rather challenging in a virtual environment. However, the PRO-Parenting team chose to mimic this tradition as closely as they could. After each session, the team would meet and discuss the strong statements that the caregiver participants made and crafted them to be a Parent Principle, which they named after the caregiver (e.g., "Lisa's Parent Principle"). These Parent Principles were then compiled and added into a Parent Principles slide in the PowerPoint that was shared each week. Utilizing the Parenting Principles in this virtual setting helps equate the online delivery to that of an in-person setting. This display of principles helps caregivers feel ownership and belonging in the group and reinforces BPT teachings. The facilitators found this to be especially important to promote group discussions which are more challenging in a telehealth format. The purpose of presenting this lesson is to call attention to the importance of making the virtual environment as similar to the in-person environment as possible. Bringing in this small component of the Incredible Years curriculum brought so much to the online group, such as liveliness, accountability, encouragement and recognition.

Next is the importance of implementing various features on the platform used for online delivery. Since Zoom was used as the platform for group sessions, the facilitators used available functions such as the whiteboard, polling feature, video sharing and breakout groups. Toggling between different functions was more stimulating than just looking at the same PowerPoint. Each of these held group members' attention better since there was a change in the form of presenting throughout the night. 
Creative incentives were another important takeaway from the virtual experience. In an in-person setting, it is easy to hand out candy or other treats for right answers, participation or presentation of strengths, but this is not so simple in a virtual setting. After some time, the facilitators found ways to call out and praise the strengths of the caregivers verbally, as well as change their Zoom name to things like "Superstar Charlie" or "Gold Star Mary" to praise and encourage their work. The use of this incentive in particular was also used to model and reinforce content delivered in the intervention. This behavior became a group norm, so much so that other caregivers began to call out gold stars for their peers, mentioning the strength and growth they were showcasing.

Another lesson is that, with permission, certain acts of personability can be very valuable. Specifically, with consent from the participants, it proved to be very satisfactory to acknowledge birthdays and other milestones in the group members' lives. Occasionally, the group even sang to people.

Last, but certainly not least, this team found great interest and effectiveness in the use of “outro music," playing part of a song at the session's end. The team member who was serving as the fidelity checker for the group at each meeting functioned as the DJ and would play between 30 seconds and a minute of an energetic and lively song at the conclusion of each session. The music caused families and facilitators to dance and be silly as they were saying goodbye for the evening. This exciting part of the group acted as a signal that it was time to go, but also left everyone on a high note while doing so. The team also chose to couple music when administering a gold star to group members as a call-out that brought that same amount of fun. 
This particular lesson was one that cast a very positive light on the cohesiveness of the group. The outro music created an opportunity for the group and facilitators to laugh, have fun, show a more human or casual side, and experience a coming together.

\section{DISCUSSION}

The PRO-Parenting family therapists have learned many things from their switch to a telehealth delivery. There have been strengths in this experience in addition to challenges that initiated conversation and action amongst the team members.

As stated earlier in this thesis, one of the most notable strengths of this experience was how quickly the team worked to transition the group intervention to an online format. The developer of the Incredible Years series, Dr. Webster-Stratton had discouraged conducting the program in a telehealth format in the past because it would not offer the same opportunities for camaraderie or connection. Despite her belief, the PRO-Parenting team saw it as an act of social justice to resume the delivery of their intervention through a virtual format because of the benefit that the continuation offered families and their communities. Based on information provided to the family therapists from the participating families, there was great satisfaction with the groups. Relating this to Dr. Webster-Stratton's blog post, the PRO-Parenting Project did not solely follow her suggested tips even though it was recommended for the Incredible Years program. The family therapists worked to provide every possible feature that exists in the in-person experience to caregivers attending their virtual groups rather than expecting to offer less content as recommended by Dr. Webster-Stratton. 
Additionally, the dynamic nature of the Incredible Years program proved to be a strength when transitioning to the virtual delivery due to its versatility. There are various components to the Incredible Years program, such as implementation of videos, breakout groups, and role playing, that offered consistent change to keep the group lively. In an online setting, this active nature was critical in maintaining the attention of caregivers.

Equally important is the knowledge and skills gained in their experience that can be applied to other facilitators or leaders that may want or have to convert their services to a virtual format. Looking back to the lessons that the family therapists compiled, there are valuable implications for future group interventions, therapy or other services. Though co-facilitation is not a requirement, it is highly recommended and beneficial. It opens up the potential for more seamless transitions between different functions or presentation formats, greater communication amongst facilitators and group members, and the ability to continue the intervention in the absence of one of the facilitators. With the help of a technical support member during the virtual sessions, issues can be troubleshooted without disruption of content delivery. This support member could be a fidelity checker, a research assistant, or another project employee that is available during the meeting times. This technical support can also help enforce the established Zoom etiquette that the group has set in place. The family therapists also noted the value in being performative on camera. This can hold true for various professions using live virtual formats, such as education and healthcare services. Utilization of a PowerPoint, while discouraged in in-person settings, was effective in the case of PRO-Parenting. It is important to use different presentation methods and platforms in this setting to convey the content. In those presentation 
methods, it is also important to add in animations and graphics that are representative of the group members' community and balance that with the words on the page so that things are relatable and not overwhelming. Practice is important, therefore additional time for planning and rehearsing the content should be carved out. The family therapists found that a planning session between the co-facilitators and later a dress rehearsal with the technical support and other team members was very effective, especially since it allowed for team members to provide their input on potential concerns the therapists had. They also saw a great deal of importance in regards to mimicking the execution of the online groups as closely to the in-person sessions as possible. This means utilizing the programs to the best of one's ability, building rapport, and working to build a true connection. This can be done with personable acts such as celebrating birthdays, incentivizing participation in ways that are constructive and so much more. When seeking an execution of these groups that is going to be most efficacious, what it really comes down to is having a team and environment that is attentive, responsive, prepared, representative and flexible.

This experience did not come without challenges, concerns and considerations. The first challenge with any group setting like this is that life does not stop or slow down around the intervention. While working with the group members over a period of time, the facilitators and members of the group develop a bond as they get to know each other more. But during this time, there are plenty of chances for other life events to occur, like job loss or changes, sickness, mental health difficulties, loss of loved ones or natural disasters. It is important to know how and be able to navigate and adapt to the stressors. For this project in particular, one of the overarching goals was stress reduction. Therefore, managing other life stressors could 
vicariously occur with the skills the caregivers learn in the PRO-Parenting Project. In projects or services where stress reduction is not a primary goal, managing stressors and troubling life events can be a challenge. There needs to be more research done regarding ways in which this can be implemented both from a facilitator and a group member standpoint.

Thinking back to the literature review article by DuPaul and colleagues, they made the assumption that the families in their study would best benefit from the asynchronous model of delivery (DuPaul et al., 2017). They made a supplemental postulation that this could potentially be contributed to the fact that there were less interactions with fellow parents of children with ADHD (DuPaul et al., 2017). The family therapists found that the use of a synchronous format strengthened the content delivery and outcome of the group. The idea here is that, for this group, it resembled the previously in-person delivery model prior to the COVID-19 pandemic. While the PRO-Parenting Project could have been conducted in an asynchronous fashion, it made the most sense to continue in a synchronous manner. An interesting direction for further research would be to develop a way to assess who asynchronous and synchronous formats work best for, as well as how they can best be used for individuals and groups. A challenge that may be present in either format is encouraging and getting group members to complete and turn in appropriate forms that are necessary for the program they are in. For the PRO-Parenting team, it was a challenging task getting all of the caregivers to complete their post-intervention evaluations from a distance. When they met in-person, it was far easier to allow the caregivers to sit in a room altogether, fill out the evaluation and turn it in upon leaving the group session. This is not always the case in a virtual group setting. This challenge may exist for other groups as well, so being able to find a solution or way to navigate it would be beneficial for all. 
Another consideration for group interventions is the favorability of having access to additional bodies to help achieve different requirements, components or goals of the group. In the PRO-Parenting Project, the switch to a telehealth setting left the childcare workers, like myself, without a task to help with. Therefore, the childcare workers were used to serve as technical support and fidelity checkers. This is not something that every group has access to, whether it be in community settings with limited resources or in groups with small staff, but it aids the delivery of the intervention immensely. Navigating the technical issues as a facilitator can be a limiting factor for the intervention. Preventing this from taking place could impact the outcomes of the group in a positive manner.

\section{FUTURE RESEARCH}

This thesis has formulated a number of directions for future research. There is a need to address how to best facilitate group discussion in an online setting. This can be a challenge even in in-person settings, so tackling this obstacle in an online setting may have implications for the in-person settings as well. Additionally, there is an unspoken, or perhaps unaddressed, assumption that everyone behaves similarly online as they would in person. There needs to be research conducted on social behaviors in virtual formats as opposed to in-person formats to see how they differ and how these potential differences may affect content delivery. Another direction for future research is to provide these groups for non-English speaking families. Making these resources accessible to these individuals would not only benefit the community in need, but also help diversify the work that these groups are doing. Speaking to the PRO-Parenting study specifically, work needs to be done in order to arrive at an understanding 
around the differing conclusions drawn by Dr. Webster-Stratton and by the PRO-Parenting Project. There needs to be a more robust understanding of what is effective and what is not so that the field is actively working to seek out the best possible ways to conduct these groups and strengthen the outcomes. Finally, the evolution of telehealth over the course of the COVID-19 pandemic needs to be assessed. As stated before, the changes that come from this point in time will impact the world far more than anyone can ever imagine. Tracking the development throughout the span of the virus will likely present valuable information that will inform many fields of study for the next foreseeable future. 


\section{REFERENCES}

Bearss, K., Burrell, T. L., Challa, S. A., Postorino, V., Gillespie, S. E., Crooks, C., \& Scahill, L. (2017). Feasibility of Parent Training via Telehealth for Children with Autism Spectrum Disorder and Disruptive Behavior: A Demonstration Pilot. Journal of Autism and Developmental Disorders, 48(4), 1020-1030. doi:10.1007/s10803-017-3363-2

Brown, S. M., Doom, J., Watamura, S., Lechuga-Pena, S., \& Koppels, T. (2020). Stress and Parenting during the Global COVID-19 Pandemic. doi:10.31234/osf.io/ucezm Coronavirus Disease (COVID-19) - events as they happen. (n.d.). Retrieved from https://www.who.int/emergencies/diseases/novel-coronavirus-2019/events-as-they-happen

Cresswell, A. (2014). Delivering Incredible Years Programme: A Practice Perspective. International Journal of Birth and Parent Education.

Dake, L. (2020, June 03). Gov. Brown Orders All Oregon Schools To Close. Retrieved from https://www.opb.org/news/article/kate-brown-orders-oregon-schools-to-close-coronavirus/

Dielman, M., Drude, K., Ellenwood, A. E., Heinlen, K., Imar, T., Lichstein, M., et al. (2010). Retrieved from. Telepsychology guidelines. Ohio Psychological Association https://www.ohpsych.org/professionalissues.aspx

DuPaul, G. J., Kern, L., Belk, G., Custer, B., Daffner, M., Hatfield, A., \& Peek, D. (2017). Face-to-Face Versus Online Behavioral Parent Training for Young Children at Risk for ADHD: Treatment Engagement and Outcomes. Journal of Clinical Child \& Adolescent Psychology, 47(Sup1). doi:10.1080/15374416.2017.1342544

Elkbuli, A., Ehrlich, H., \& Mckenney, M. (2020). The effective use of telemedicine to save lives 
and maintain structure in a healthcare system: Current response to COVID-19. The American Journal of Emergency Medicine. doi:10.1016/j.ajem.2020.04.003

Freud, S. (1955). Standard edition of the complete psychological works of Sigmund Freud. Volume x (1909): Two case histories ('Little Hans” and the “Rat Man'). London: Hogarth Press.

Heid, M. (2020, May 07). Mental Distress Among Americans Has Tripled During Pandemic. Retrieved from https://time.com/5833619/mental-health-coronavirus/

Mcintyre, L. L. (2008). Adapting Webster-Stratton's incredible years parent training for children with developmental delay: Findings from a treatment group only study. Journal of Intellectual Disability Research, 52(12), 1176-1192.

doi:10.1111/j.1365-2788.2008.01108.x

McIntyre, L.L., Neece, C. L. (2018) PRO-Parenting Research Plan.

Multnomah County situation updates on COVID-19. (2020, November 12). Retrieved from https://multco.us/novel-coronavirus-covid-19/multnomah-county-situation-updates-covid19

National Association of Social Workers (NASW). (n.d.). Retrieved from https://www.socialworkers.org/About/Ethics/Code-of-Ethics/Code-of-Ethics-English

Neece, C., Mcintyre, L. L., \& Fenning, R. (2020). Examining the impact of COVID-19 in ethnically diverse families with young children with intellectual and developmental disabilities. Journal of Intellectual Disability Research. doi:10.1111/jir.12769

Nirmita Panchal, R. K., \& 2020, A. (2020, August 21). The Implications of COVID-19 for Mental Health and Substance Use. Retrieved from 
https://www.kff.org/coronavirus-covid-19/issue-brief/the-implications-of-covid-19-for-me ntal-health-and-substance-use/

Oregonian/OregonLive, H. B. (2020, July 23). Oregon coronavirus restrictions: Gov. Kate Brown issues new rules on businesses as state struggles to control spread. Retrieved from https://www.oregonlive.com/news/2020/07/oregon-gov-kate-brown-announces-new-maskmandate-for-kids-earlier-bar-and-restaurant-closure-times.html

Perle, J. G., Langsam, L. C., \& Nierenberg, B. (2011). Controversy clarified: An updated review of clinical psychology and tele-health. Clinical Psychology Review, 31(8), 1247-1258. doi:10.1016/j.cpr.2011.08.003

Taylor, T. K., Webster-Stratton, C., Feil, E. G., Broadbent, B., Widdop, C. S., \& Severson, H. H. (2008). Computer-Based Intervention with Coaching: An Example Using the Incredible Years Program. Cognitive Behaviour Therapy, 37(4), 233-246. doi:10.1080/16506070802364511

Telehealth Continuing Education Resources. (n.d.). Retrieved from https://www.apa.org/ed/ce/telehealth

VanderHart, D. (2020, June 03). Gov. Kate Brown Issues Order Directing Oregonians To 'Stay Home'. Retrieved from https://www.opb.org/news/article/oregon-stay-at-home-order-coronavirus-covid-19-kate-b rown/

Webster-Stratton, C. (2001). The Incredible Years: Parents, Teachers, and Children Training Series. Residential Treatment for Children \& Youth, 18(3), 31-45. doi:10.1300/j007v18n03_04 
Webster-Stratton, C. (2020, October 20). The Incredible Years ${ }^{\circledR}$ Blog. Retrieved November 29, 2020, from https://incredibleyearsblog.wordpress.com/ 